\title{
Dense-phase velocity fluctuation in a 2-D fluidized bed
}

\author{
S. Sánchez-Delgado *, C. Marugán-Cruz, A. Acosta-Iborra, D. Santana \\ Departamento de Ingeniería Térmica y de Fluidos, Universidad Carlos III de Madrid, Avda. de la Universidad 30, 28911 Leganés, Madrid, Spain
}

\begin{abstract}
A B S T R A C T
This work presents an investigation of the perturbations induced by the bubbles in a 2 D fluidized bed. A combination of Digital Image Analysis (DIA) and Particle Image Velocimetry (PIV) techniques was developed to characterize the dense and bubble phases. The analysis of the mean and the instantaneous fluctuations of the velocity of the dense phase, together with the solid movement around bubbles, allowed for the measurement of the influence region, distinguishing an upward moving dense phase in the nose and the wake of the bubble (drift) and a downward moving dense phase in the sides of the bubble.

For an isolated bubble, we measured the drift area within the total influence region and related the size of these regions to the equivalent diameter of the bubble. This work also presents results on the volumetric dissipation of kinetic energy, where we concluded that the energy dissipation in the dense phase is proportional to the square of the bubble velocity.
\end{abstract}

\section{Introduction}

Understanding the processes involved in the mixing of solids is essential for the design of fluidized bed reactors. In 1954, Lacey distinguished three components in a mixing process: 1) convective, 2) diffusive and 3) shear mixing [1]. Generally, these mechanisms are combined in most processes, although in some processes one of them predominates. Convective mixing mechanism involves the transfer of a group of particles from one location to another, diffusive mixing involves the random movement of particles and shear mixing involves the formation of slip planes within the mixture.

Later, Shen et al. presented an investigation of the dynamics of solids mixing in fluidized beds [2]. Their mechanistic model of a gas solid fluidized bed was based on the models of Kunii and Levenspiel [3] and Fan et al. [4]. This model assumed that the fluidized bed consists of three phases, a dilute phase containing no solids (the bubble phase), an upward moving dense phase (the wake phase) and a downward moving dense phase (the emulsion phase). To solve the convection diffusion equation, they used a power law difference method; the numerical results were in satisfactory agreement with the previously existing experimental data.

Recently, Lu and Hsiau [5] employed a Discrete Element Method (DEM) to simulate the motion and mixing behavior of granular materials in three dimensional vibrated beds. In their work, a study of the importance of the mixing mechanisms is also presented, using the

\footnotetext{
* Corresponding author. Tel.: + 34 916246223; fax: + 34916249430.

E-mail address: ssdelgad@ing.uc3m.es (S. Sánchez-Delgado).
}

index, M, defined by Lacey [1] as the parameter to quantify the mixing quality.

Digital Image Analysis (DIA) and Particle Image Velocimetry (PIV) are two of the most common techniques applied to $2 \mathrm{D}$ fluidized beds to analyze the bubble and emulsion phases. $2 \mathrm{D}$ fluidized beds allow for bubble visualization, making it possible to obtain relevant bubble parameters (center of mass, velocity, equivalent diameter, etc.) to characterize the bubbling behavior of the fluidized bed. Additionally, the emulsion phase velocity can be characterized using the PIV technique. Recently, Sanchez Delgado et al. [6] studied the time averaged bubble concentration and time averaged particle velocity in a $2 \mathrm{D}$ fluidized bed to characterize the behavior of fluidized beds with different bed aspect ratios and superficial gas velocities. These techniques were applied by Almendros Ibáñez et al. [7] to study the different mechanisms for solid ejection in gas fluidized beds. Busciglio et al. [8] presented a digital image analysis technique developed to study the fluidization dynamics of a lab scale bubbling bed. They compared the main bubble parameters (bubble size and bubble velocity distribution) with correlations presented in the literature and confirmed the applicability of the DIA technique. Laverman et al. [9] combined the DIA and the PIV techniques to study the bubble behavior and to characterize the emulsion phase. Specifically, they studied the influence of particles raining through the bubble on the time averaged velocity profile of the emulsion phase.

In this work, the perturbation region generated by a bubble in a $2 \mathrm{D}$ fluidized bed has been studied using the time averaged emulsion velocity field and the calculation of fluctuating velocity component of the dense phase as proposed by Liu et al. [10]. Applied to fluidized beds, this technique is able to establish the region of the dense phase where the effect of the bubble rise is important in terms of solids 
mixing. Furthermore, for an isolated bubble, it is possible to identify two phases containing the entire solid: the upward moving dense phase in the wake of the bubble and the downward moving dense phase at the sides of the bubble. The experimental results are in satisfactory agreement with the mechanistic model proposed by Shen et al. [2].

Although the attrition phenomena depend on many factors, such as the physical chemical nature of the particles and the process conditions, one fundamental variable related to fluidized bed dynamics is the shear between particles [11]. With the results obtained using the proposed techniques, valuable information about the shear can be obtained to improve attrition modeling in fluidized beds.

In this work, the application of non intrusive techniques in a $2 \mathrm{D}$ fluidized bed allows to calculate the total influence region in the dense phase, $A_{t}$, and the size of the drift region, $A_{d}$, within the total influence region.

In addition, a parameter, $\sigma$, is defined as the ratio of the volumetric dissipation of kinetic energy and the apparent viscosity. It will be demonstrated that this parameter is proportional to the square of the bubble velocity, $U_{b}^{2}$. Therefore, the volumetric dissipation of kinetic energy in the dense phase can be related to the kinetic energy of the bubble. This relation represents an initial quantification of bubble kinetic energy in the attrition phenomena in fluidized beds.

\section{Experimental setup}

Fig. 1 shows a schematic diagram of the image acquisition system and the fluidized bed apparatus employed in the experiments. The experiments were carried out in a $2 \mathrm{D}$ cold fluidized bed $50 \mathrm{~cm}$ wide $(w), 200 \mathrm{~cm}$ high $(h)$ and $0.5 \mathrm{~cm}$ thick $(t)$. The front wall was made of glass whereas the back panel was made of aluminum and covered by a black card to obtain a higher contrast in the images. Two $650 \mathrm{~W}$ spotlights were used to provide uniform illumination. The bed was filled with a mixture of black and white Geldart B glass particles [12], with a density, $\rho_{p}$, of $2,500 \mathrm{~kg} / \mathrm{m}^{3}$ and a diameter, $d_{p}$, of $600800 \mu \mathrm{m}$, previously sieved, following a normal distribution, with a mean and standard deviation of $677.8 \mu \mathrm{m}$ and $93.3 \mu \mathrm{m}$, respectively. The ratio between the superficial gas velocity, $U_{g}$, and the minimum fluidization velocity, $U_{m f}$, was equal to $1.75\left(U_{m f}=35 \mathrm{~cm} / \mathrm{s}\right)$.

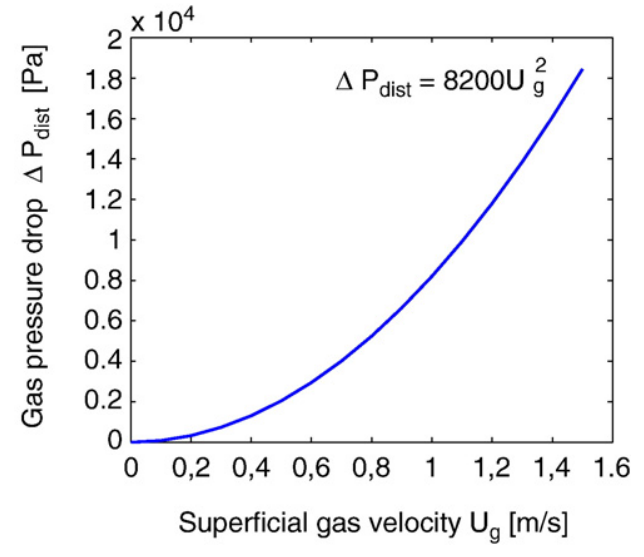

Fig. 2. Characteristic curve of the air distributor.

Only the left side of the fluidized bed was recorded due to the symmetric configuration of the bed; the imaged area was: $h_{1} \times w_{1}=30 \mathrm{~cm} \times 25 \mathrm{~cm}$ and the images were recorded at a frame rate, $f_{a}$, of $125 \mathrm{fps}$ with a camera resolution of $1270 \times 992$ pixels.

The air distributor consisted of a perforated plate with 50 holes of $1 \mathrm{~mm}$ diameter spaced $1 \mathrm{~cm}$ apart. The characteristic curve of the air distributor is shown in Fig. 2

During the experiments, the gas pressure drop through the bed was $\triangle P \sim 4500 \mathrm{~Pa}$ and the mean superficial gas velocity was around $0.62 \mathrm{~m} / \mathrm{s}$. Therefore, the distributor and the bed pressure drops during experi ments were of the same order. Under these operating conditions the bed and the air supply system are independent [13 15].

In this work, a special PIV code, MatPIV 1.6.1 [16], has been used to obtain the dense velocity field. Interrogation windows of $16 \times 16$ pixels with a $50 \%$ overlap were typically used in the PIV analyses performed to compute the velocity fields of the dense phase. Recently, several authors have used this application on $2 \mathrm{D}$ fluidized beds to calculate particle velocities $[7,17]$. For these experiments, the fluidized bed column was filled with a total weight of $1600 \mathrm{~g}, 1568 \mathrm{~g}$ of white particles and $32 \mathrm{~g}$ of black ones to improve the measuring technique [17].

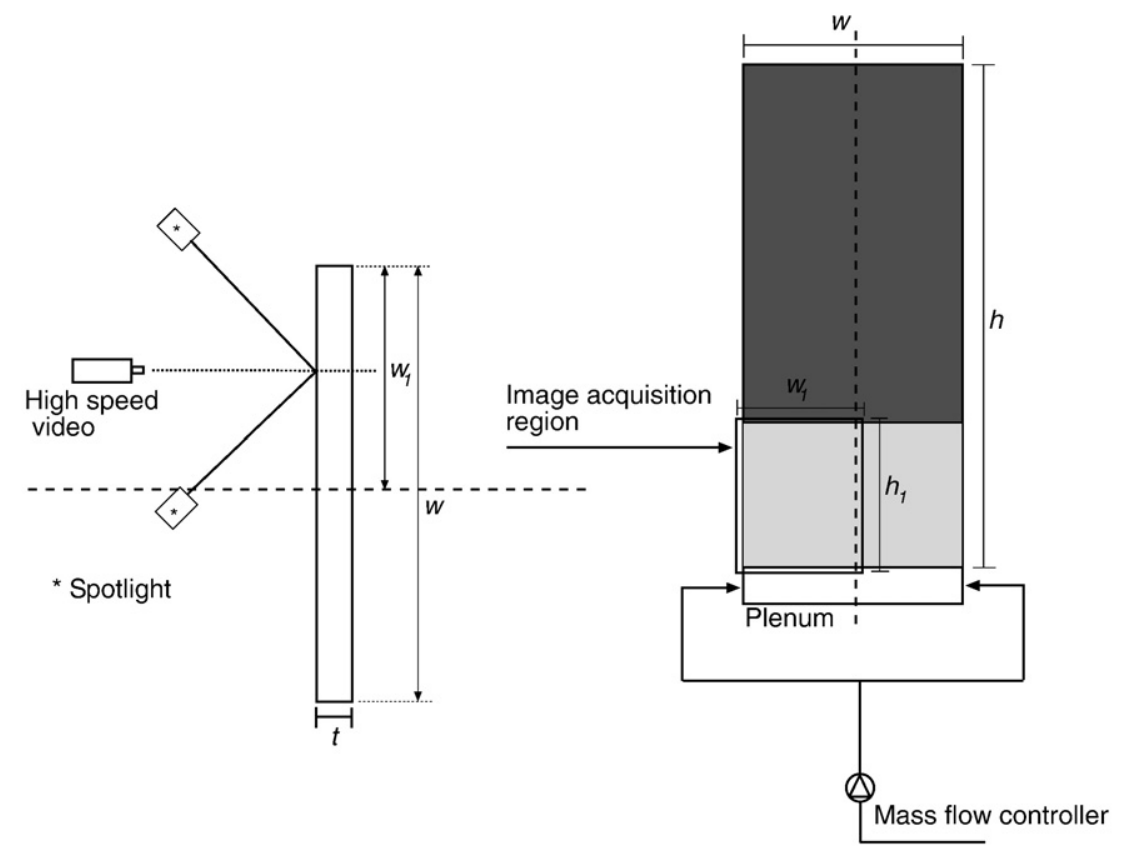

Fig. 1. Experimental setup. 
a

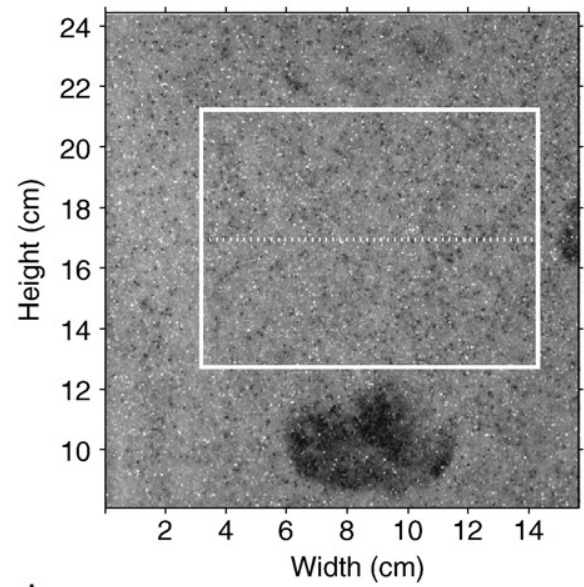

d

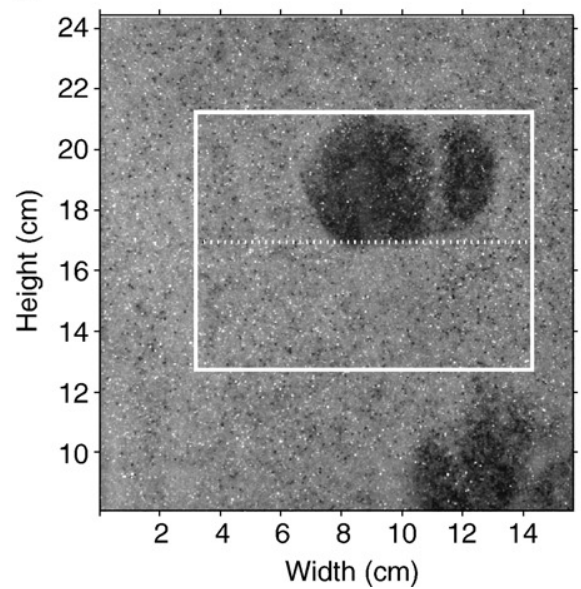

b

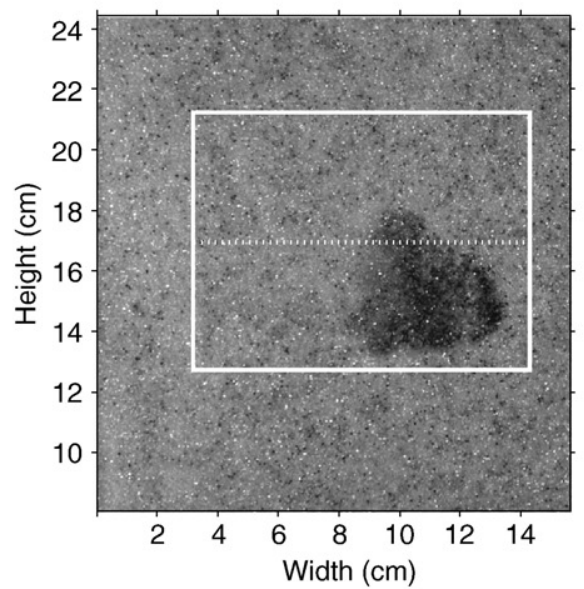

e

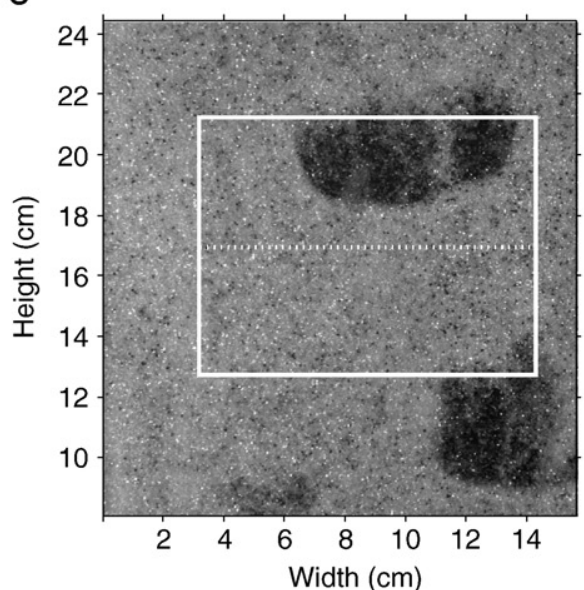

C

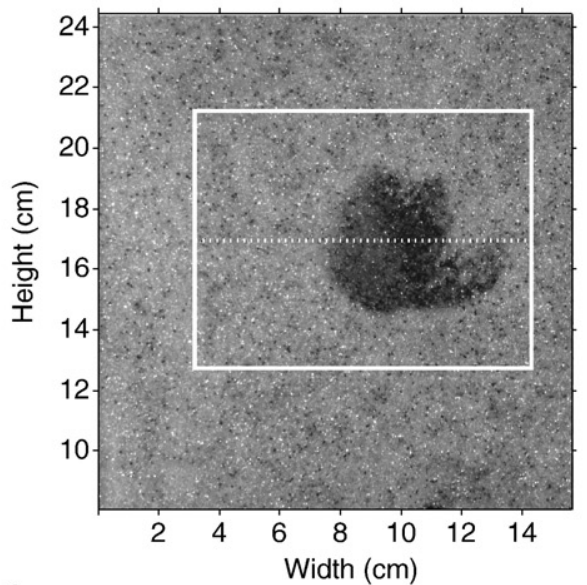

f

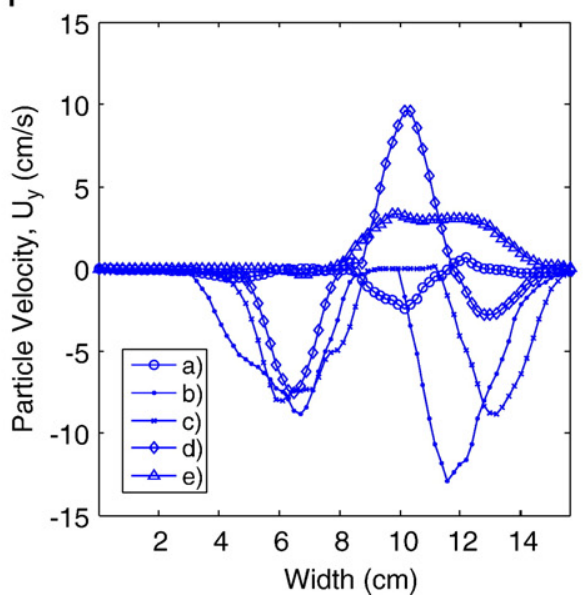

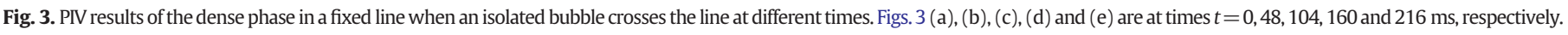

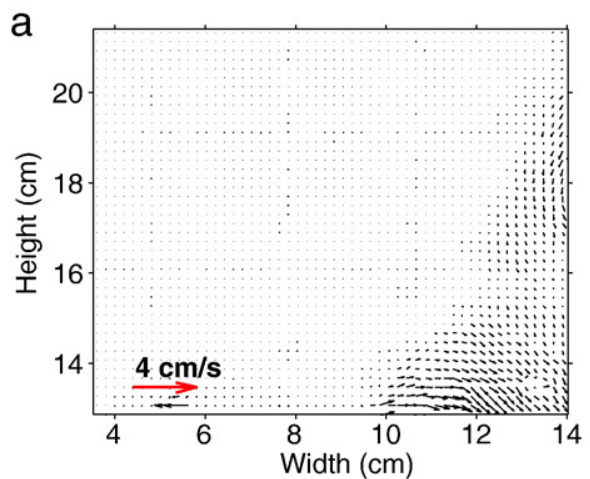

d

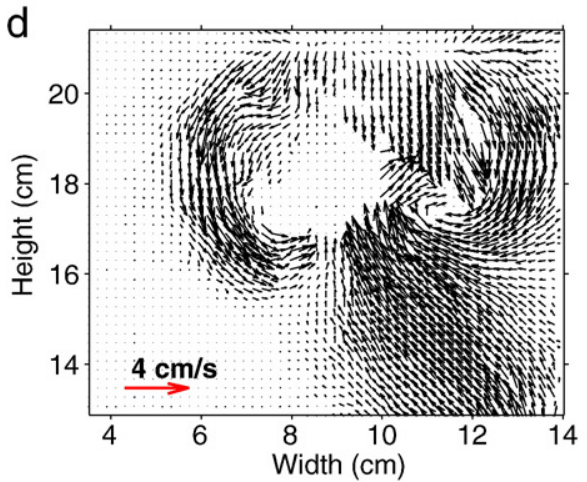

b
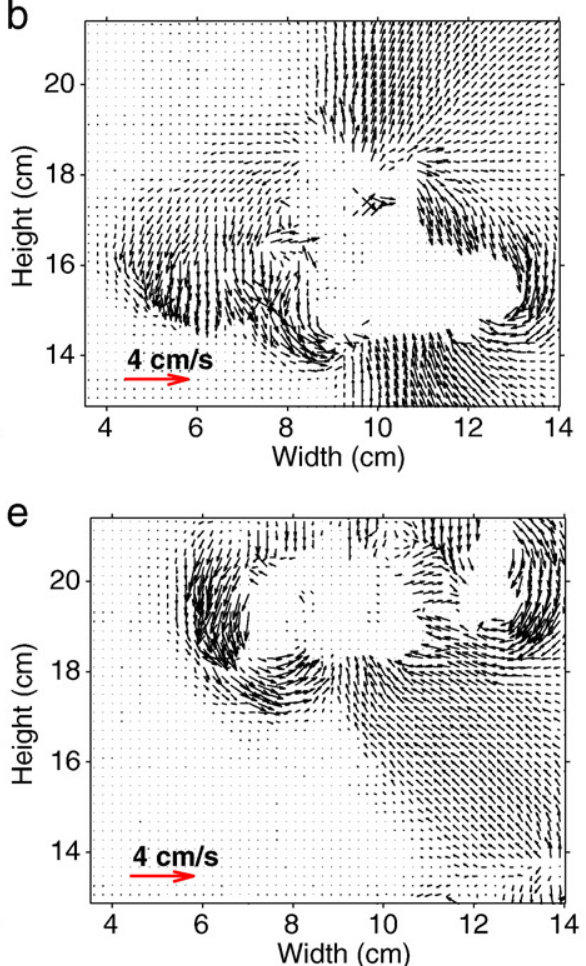
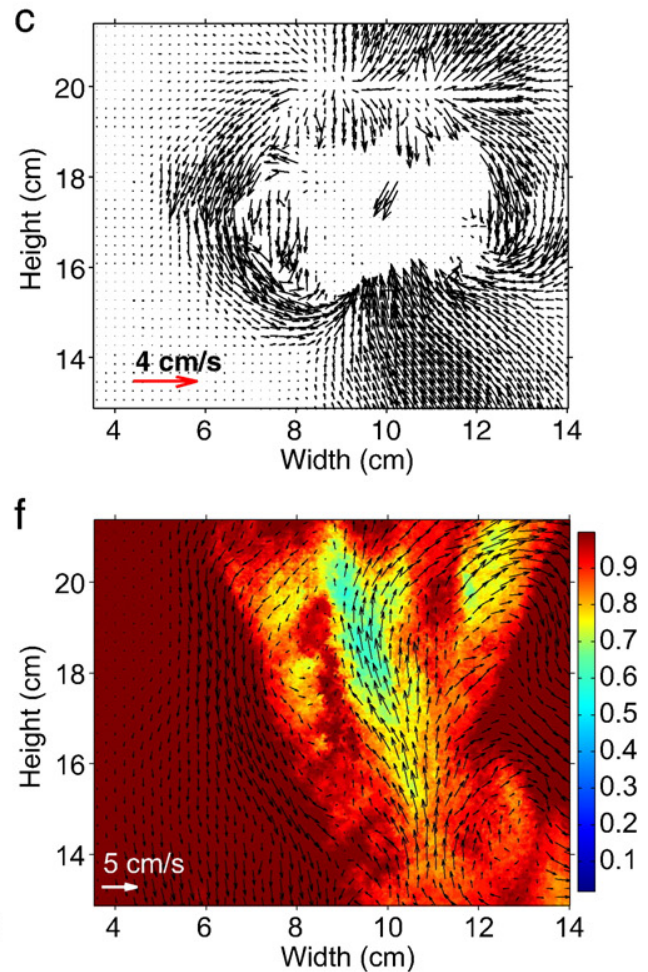

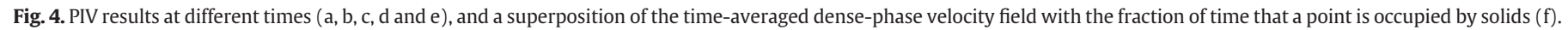


DIA was applied to establish the boundaries between the dense phase and the bubble phase. The analysis was based on the calculation and application of a threshold level over each image [18]. Using this technique, not only are the phases clearly identified (dense phase and bubble phase), but also different bubble properties could be calculated, such as the equivalent diameter based on bubble area, $D_{e q}=\sqrt{\frac{4 A_{b}}{\pi}}$, the mass center or the bubble velocity [19].

\section{Results and discussion}

During the rise of a single bubble, it is possible to identify solid particles that are influenced by that motion. When a pair of bubbles is rising vertically, the trailing bubble is accelerated under the influence of the leading bubble; eventually, the dome of the trailing bubble enters into the wake of the leading bubble and they become a single bubble $[7,20]$. During coalescence, it is difficult to establish the influence region of each bubble.

\subsection{Bubble induced particle velocity}

Typically, when a bubble is traveling toward the top of the bed, the particles in the dense phase can either rain through the bubble or move downward, surrounding the bubble. The particles located in the wake of the bubble are accelerated due to the wake effect on the dense phase. Applying PIV techniques to the recorded images, we can obtain the variations of the dense phase velocity.

Snapshots of the bubbling bed are displayed in Figs. 3(a) (e). In these figures, the rectangular region and the horizontal line were drawn to show where the calculations were performed. We defined $t=0$, Fig. 3(a), as the instant of time when the bubble enters the study region. Fig. 3(f) shows the vertical velocity of the dense phase, $U_{y}$, at the horizontal line for those snapshots.

When a bubble reaches the horizontal line (Figs. 3 (b) (c)) the dense phase has a downward movement around the bubble. Fig. 3(d) shows the instant of time at which the wake of the bubble is crossing the line; it can be observed that the solids in this region have an upward motion.
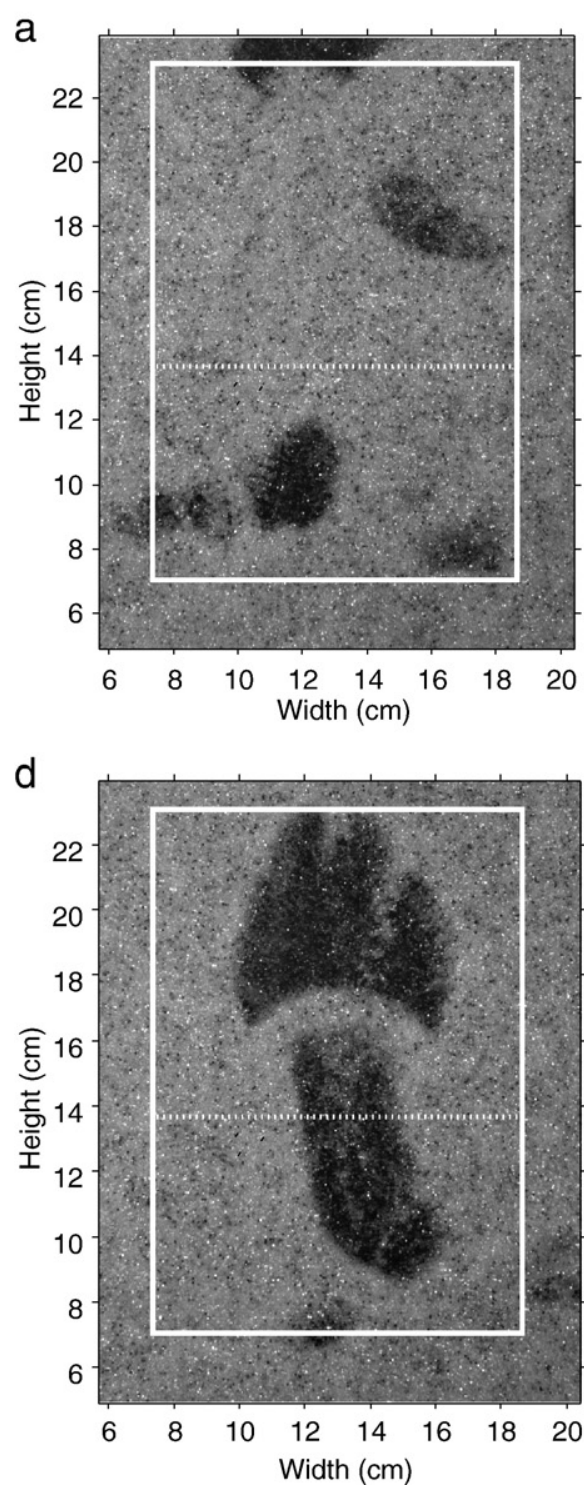
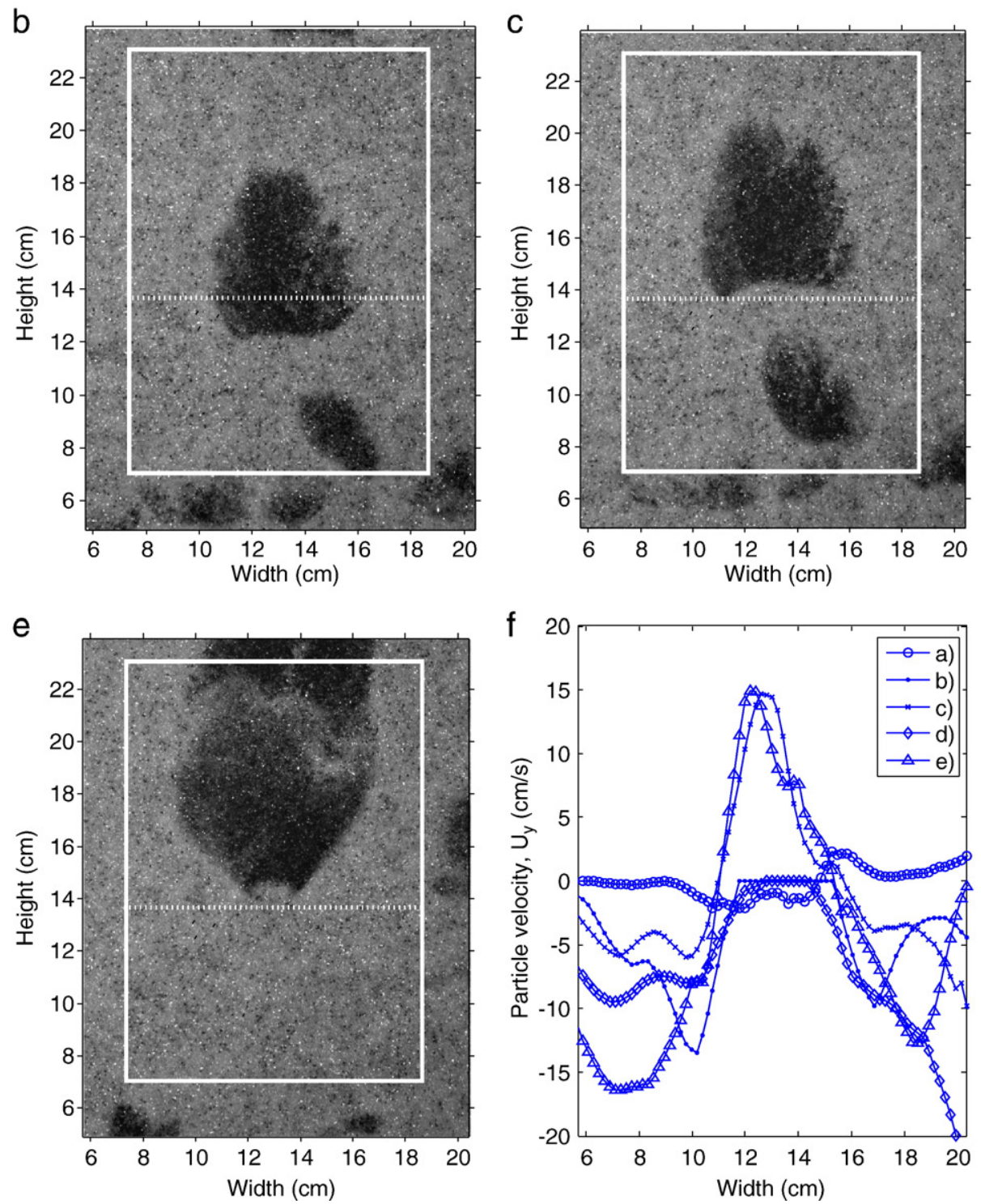

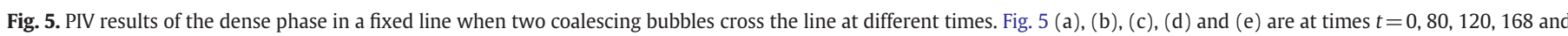
$256 \mathrm{~ms}$, respectively. 
Considering $T$ as the time interval between $t=0$ and the moment at which the bubble leaves the study region, the time averaged dense phase velocity fields can be calculated as follows:

$\overline{U(\boldsymbol{x})}=\left(\sum_{n=1}^{N} U_{n}(\boldsymbol{x}, t)\right) / N$

where $N$ is the number of images corresponding to the time interval considered to calculate the average velocity $\left(N=f_{a} \cdot T\right)$.

As stated above, a threshold level was calculated to transform the grayscale image into black and white to aid in distinguishing the dense phase and the bubble phase [18]. For each image, the pixels representing the dense phase are given a value of $1\left(C_{n}(\boldsymbol{x})=1\right)$ and the pixels representing the bubble phase are given a value of
$0\left(C_{n}(\boldsymbol{x})=0\right)$. Then, the fraction of time that a point, $\boldsymbol{x}$, is occupied by solids can be calculated as follows:

$\overline{C(\boldsymbol{x})}=\frac{\sum_{n=1}^{N} C_{n}(\boldsymbol{x})}{N}$

For the images analyzed in Fig. 3, the velocity fields in the selected region are represented in Figs. 4 (a), (b), (c), (d) and (e). Additionally, Fig. 4 (f) represents a superposition of $\bar{C}$ (the color map) and the time averaged velocity of the dense phase.

Figs. 4 (b) (e) show the effect of an isolated bubble on the dense phase. It can be seen that the particles located at the sides of the bubble move downward and the particles in the wake of the bubble move upward. The time averaged velocity field of the dense phase, $\bar{U}$, a

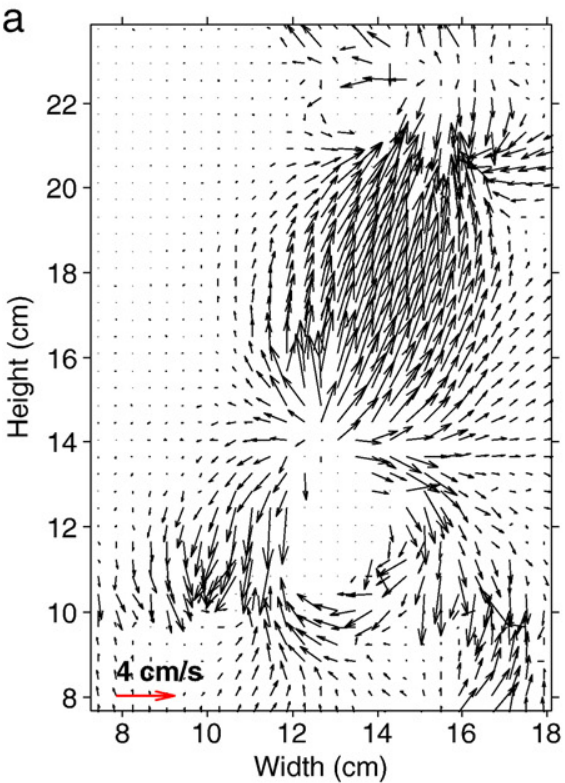

d

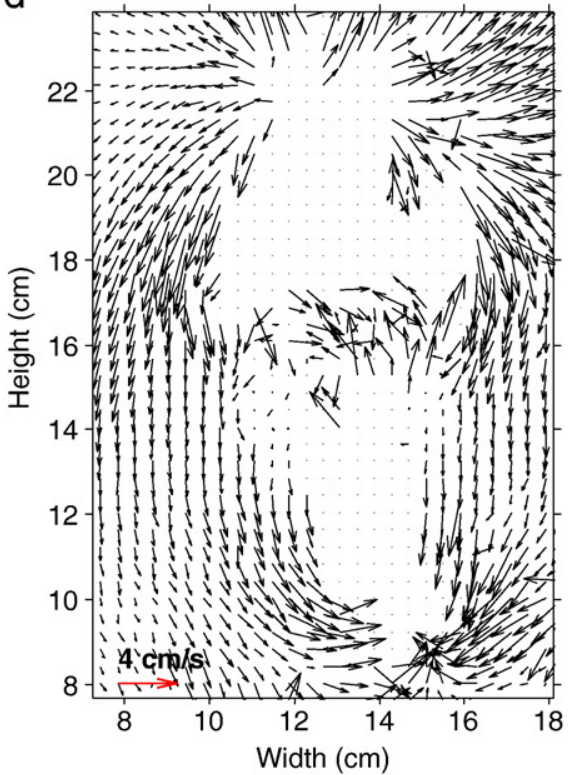

b

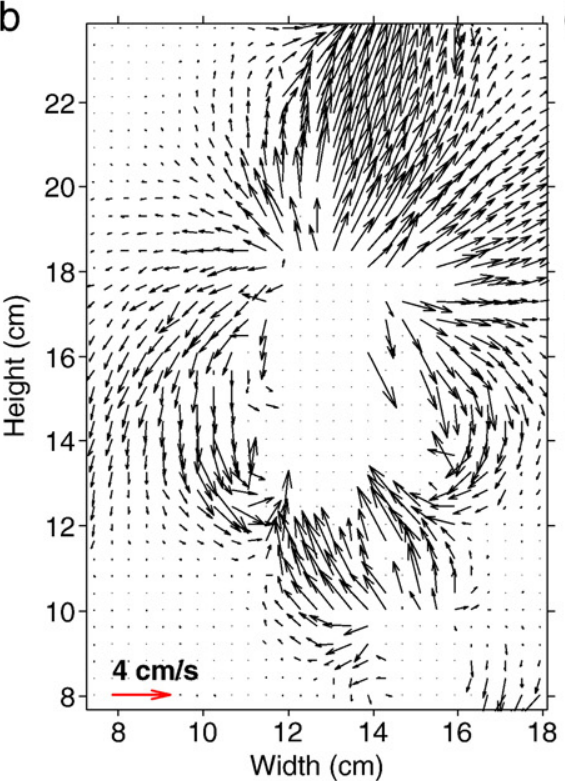

e

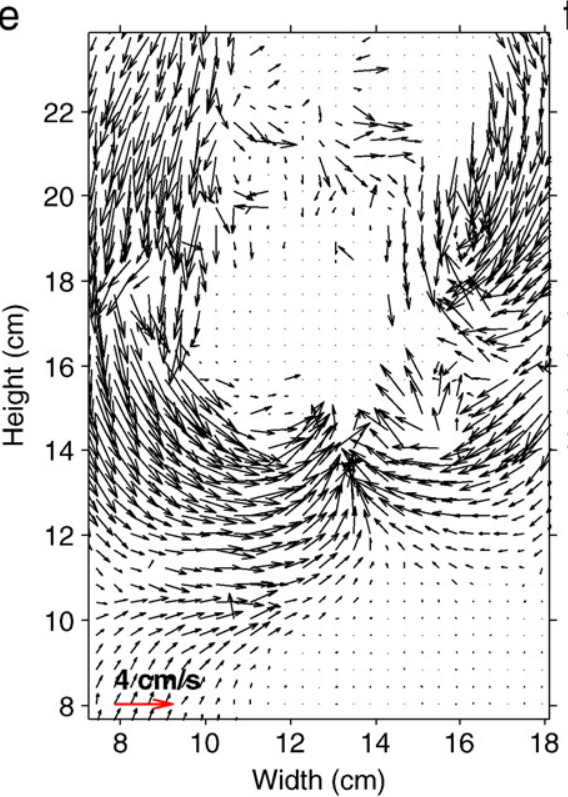

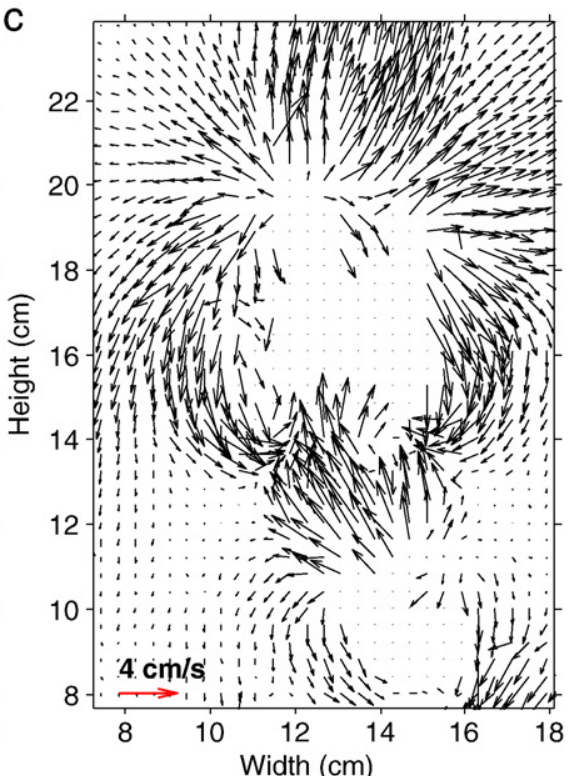

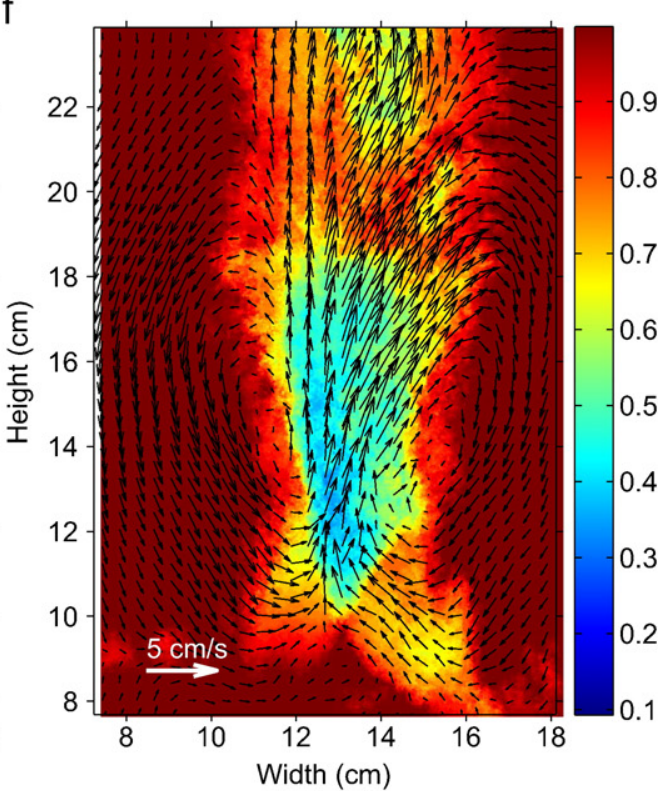

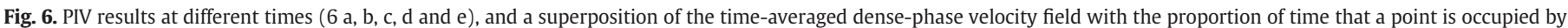
solids (f) for an isolated bubble. 
together with the fraction of time that a point is occupied by solids, $\overline{C(\boldsymbol{x}})$, are represented in Fig. 4 (f). Taking a closer look at this figure, three different zones can be distinguished in terms of solids transport mechanisms: (i) a region that is always occupied by solids $(\bar{C}=1)$, where the dense phase is moving downward, (ii) an upward moving dense phase with the lowest values of $\bar{C}$, and (iii) an interface layer between these two regions, where vortices appear. According to Shen et al. [2], solid mixing is composed of two components: the first is the convection component between the upward and the downward moving regions of the dense phase and the second is the diffusion component in the downward dense phase.

For the same fluidization conditions, $U_{g} / U_{m f}=1.75$, the same procedure was performed to analyze the bubble coalescence phe nomenon (Fig. 5).

The velocity fields shown in Figs. 6 (a) (e) correspond to the flow visualizations presented in Figs. 5(a) (e). Fig. 6 (f) shows a super position of the fraction of time that a point is occupied by the emulsion phase and the time averaged dense phase velocity field.

In this case, it is also possible to distinguish the three regions of the solids mixing mechanism described above. It can be observed that the interface layer for the coalescing bubble is narrower than for the case of the isolated bubble. Furthermore, due to this coalescing effect, the velocities in the dense phase reach higher values and the effect of the two wakes increase the solids transport.

\subsection{Bubble influence and dense phase velocity perturbation}

Once the effect of a rising bubble on the dense phase has been characterized, it is important to quantify the size of the region in the dense phase where the effect of the bubble rise is important in terms of solids mixing (i.e., the influence region).

In this section, the dense phase velocity perturbation is analyzed. With this aim, the absolute fluctuating velocity, $\left|U^{\prime}\left(\boldsymbol{x}, t_{n}\right)\right|$, was obtained by:

$\left|U^{\prime}\left(\boldsymbol{x}, t_{n}\right)\right|=\left|U\left(\boldsymbol{x}, t_{n}\right)-\bar{U}(\boldsymbol{x})\right|$
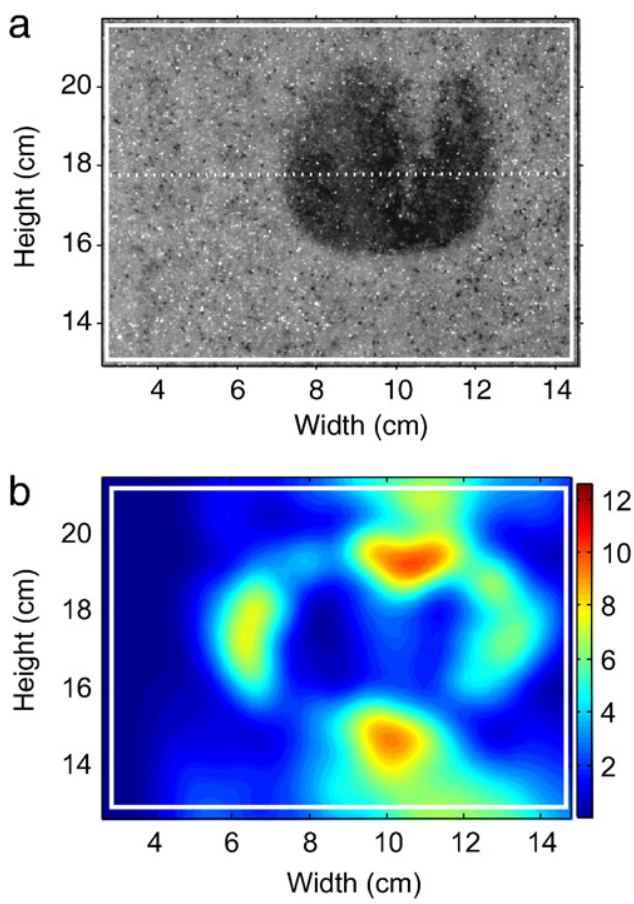

Fig. 7. (a) Flow visualization of a bubble; (b) Absolute vertical velocity perturbation of the dense phase.

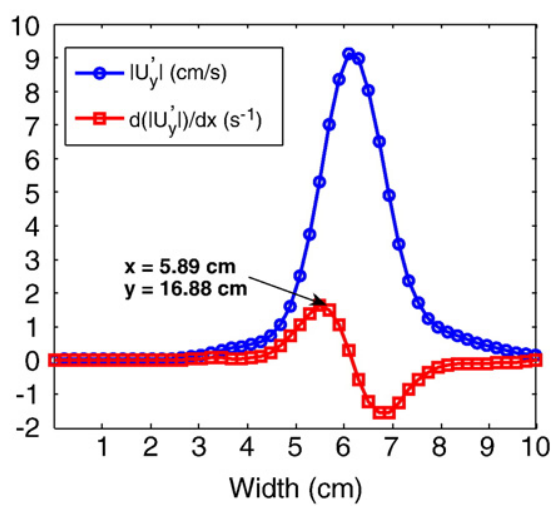

Fig. 8. Absolute velocity perturbation of the dense phase, and horizontal derivative along the line of Fig. 7(a).

Fig. 7(a) shows a snapshot of a bubble moving upward in our $2 \mathrm{D}$ bed. The absolute fluctuation velocity of the emulsion region was calculated at that instant of time (Fig. 7(b)).

Three regions can be identified surrounding the bubble. In the dense phase at the top of the bubble, a region of high magnitude velocity perturbation appears due to the effect of the nose of the bubble. A second region can be distinguished where the particles move upward, affected by the wake of the bubble, and finally at the bubble sides, two zones of high absolute perturbation values are identified, corresponding to the downward moving dense phase. This visual inspection is complemented with a numerical analysis to define the influence region of a bubble rising over the dense phase. Fig. 8 represents the absolute velocity perturbation along the horizontal line defined in Fig. 7 (a) as well as the horizontal derivative of that magnitude.

In this analysis, the perturbation values denote, quantitatively, the influence of a rising bubble and the derivate of the perturbation provides information about the bubble influence region.

The position where the derivative reaches a maximum is considered as the boundary of the influence region (marked by black dots in Fig. 9), and therefore the value of $\left|U^{\prime}(\boldsymbol{x})\right|$ at this position would be the threshold value designated to establish that the dense phase is affected by the bubble. Fig. 10(a) shows the points at which the dense phase velocity perturbations are larger than the threshold value previously calculated.

Fig. 10 (b) shows the two emulsion phases defined according to the direction of movement: an upwardly moving dense phase in the bubble wake dominated by convective mechanisms and a down wardly moving dense phase where the diffusion effect is the most relevant mixing mechanism [2]. These regions are in accord with the influence regions plotted in Fig. 10 (a).

The same analysis applied to an isolated bubble has been applied for two coalescing bubbles. Fig. 11 shows the flow visualization of two

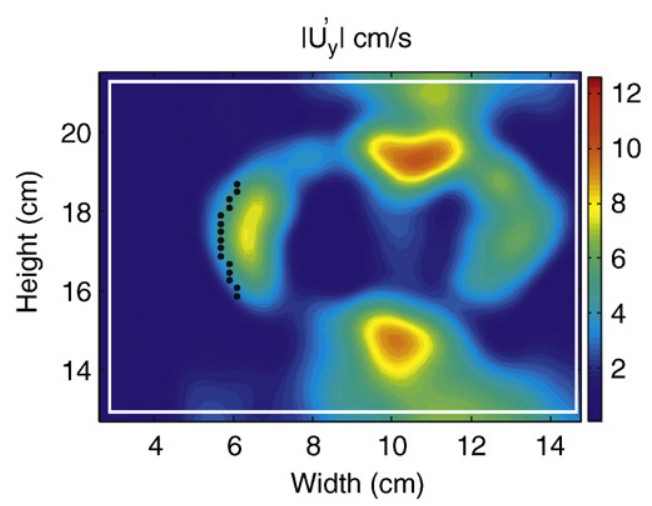

Fig. 9. Boundary of the influence region at the left side of the bubble shown in Fig. 7(a) 


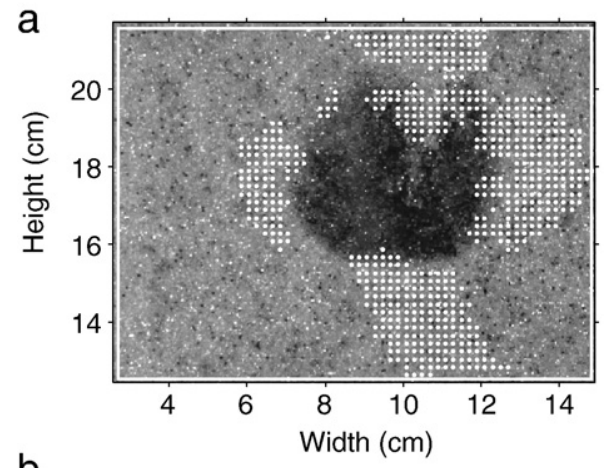

b

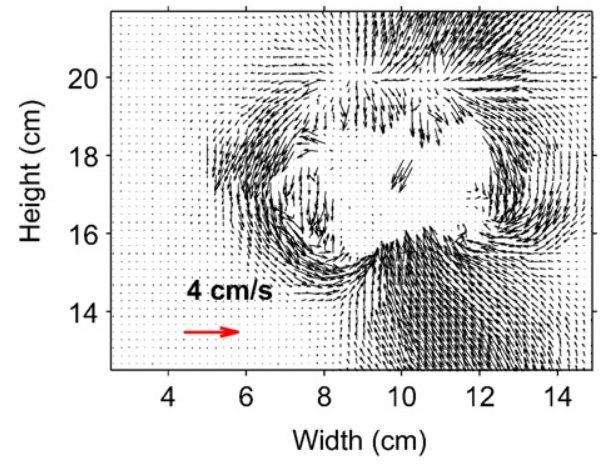

Fig. 10. (a) Representation of the influence region over the original image; (b) PIV results for Fig. 10a.

coalescing bubbles, the absolute vertical velocity perturbation of the dense phase, $\left|U_{y}^{\prime}(x)\right|$, and the total influence region generated by the bubble chain. This analysis does not allow for the distinction of the influence region generated by each bubble.

\subsection{Influence region measurement and dissipation of kinetic energy}

In this section, different bubble parameters were calculated and compared for a total of 20 isolated bubbles: equivalent bubble diameter, $D_{e q}$, center of mass, $\boldsymbol{x}_{c m}$, bubble velocity, $U_{b}$, influence region, $A_{t}$, and drift

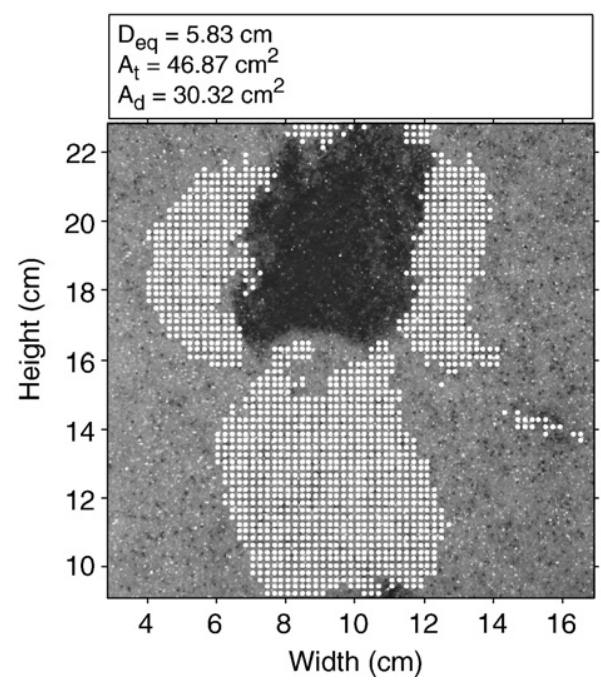

Fig. 12. Qualitative results for an isolated bubble.

region, $A_{d}$. Fig. 12 shows an example of the calculation of these parameters for one bubble.

Fig. 13 (a) shows the relationship between the total influence region and the perturbation region occupied by the wake. A linear regression fitting was applied to these data. It can be seen that the drift area represents around 50\% of the influence region. Fig. 13 (b) shows the relationship between the total influence region and the equivalent diameter of the bubble. As expected, larger bubbles generate larger influence regions, and a linear relationship between total influence region and the equivalent square diameter, $A_{t} \propto D_{\text {eq }}^{2}$, was observed; Fig. 13 (c) also shows a linear relationship between the drift area and the equivalent diameter, $A_{d} \propto D_{e q}$.

In a bubbling fluidized bed, the mechanical power supplied by the air system is given by the product of the volumetric flow rate of gas times the pressure drop. This input energy can be divided into two terms: the energy to keep the particles in suspension and the input energy that remains for bubble formation, and thus for attrition [11]. Attrition promoted by bubbles takes place in regions where there are severe gradients in the dense phase velocity. Under the model of a

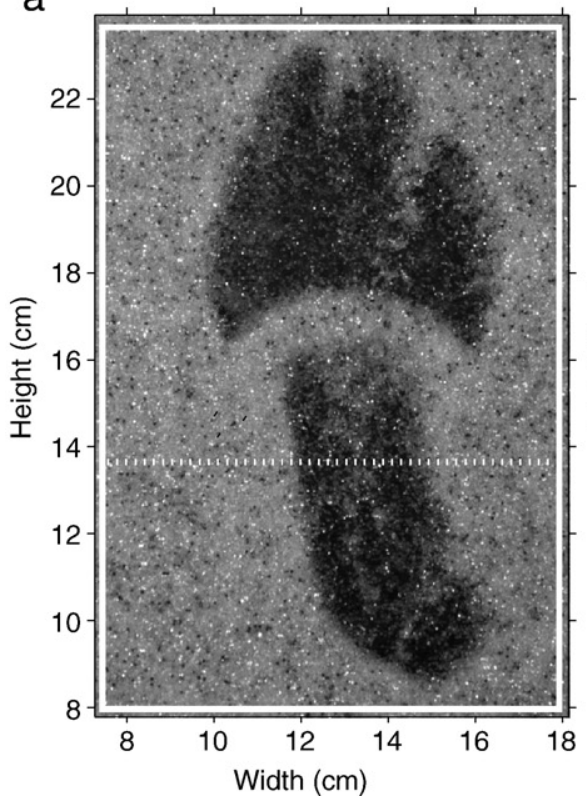

b

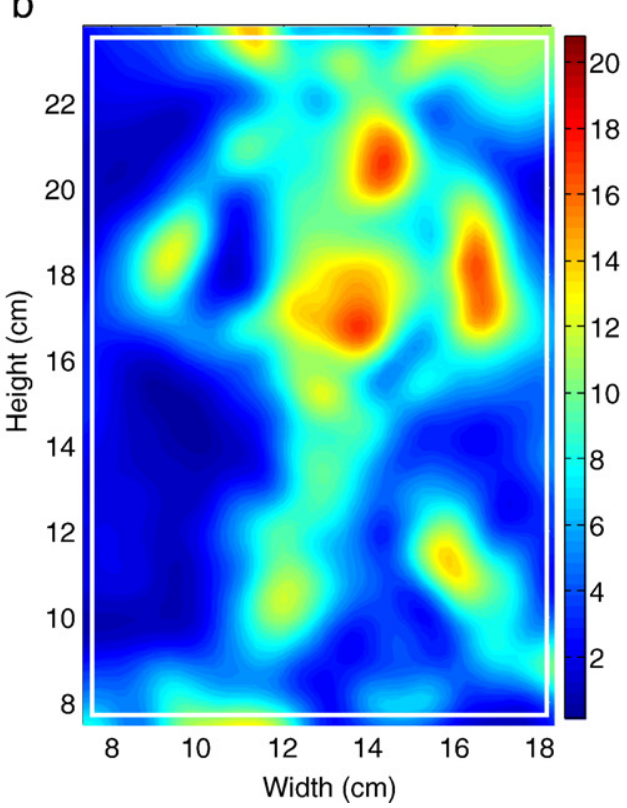

C

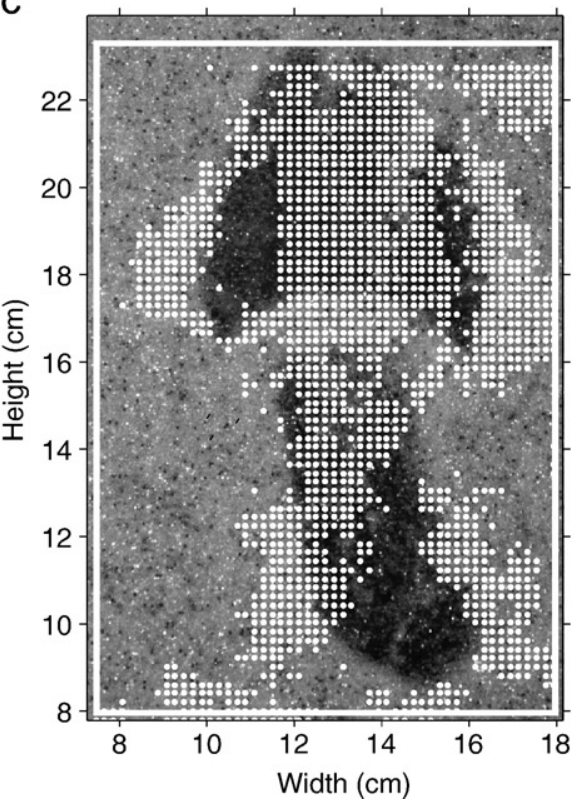

Fig. 11. (a) Coalescing bubbles in a 2-D fluidized bed. (b) Absolute velocity perturbation of the dense phase. (c) Total influence region generated by the bubbles. 


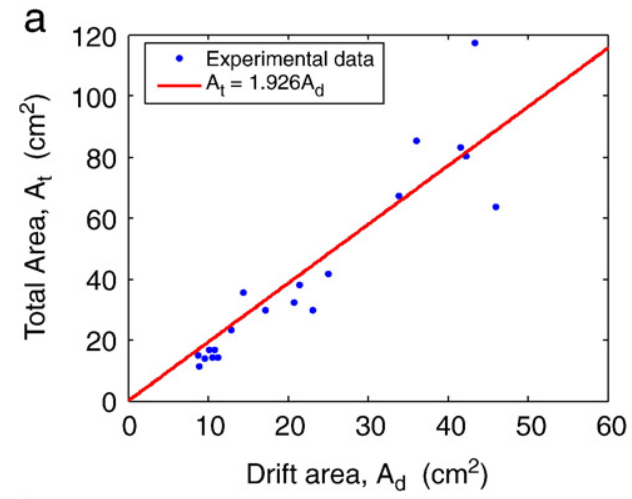

b

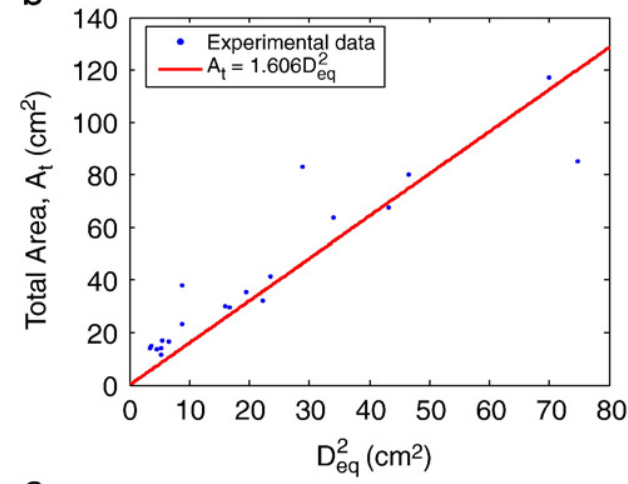

C

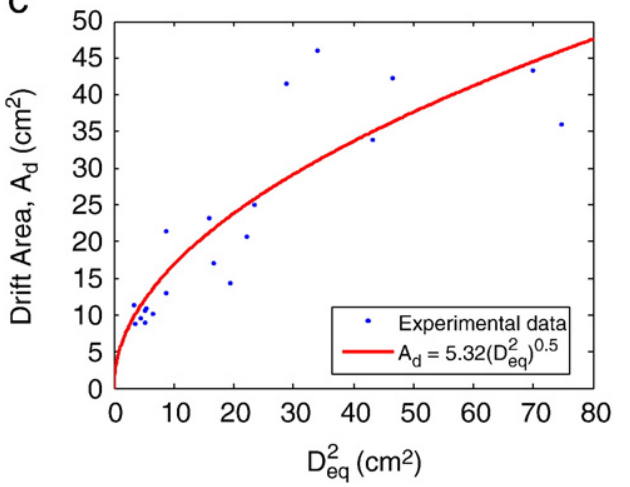

Fig. 13. Experimental relationship between bubble size and influence-region size: (a) Total influence region and drift size; (b) Total influence region and bubble equivalent diameter squared; (c) Drift area and bubble equivalent diameter squared.

dense phase behavior as an incompressible fluid, attrition can be interpreted as a mechanism of particle kinetic energy dissipation that results in the fracture or degradation of particles. The energy equation shows that dissipation is related to the particle velocity gradients along the $x$ and $y$ directions:

$\sigma=\frac{\Phi_{v}}{\mu}=\left[2\left(\frac{\partial u}{\partial x}\right)^{2}+2\left(\frac{\partial v}{\partial y}\right)^{2}+\left(\frac{\partial u}{\partial y}+\frac{\partial v}{\partial x}\right)^{2}\right]$

where $\Phi_{v}$ is the volumetric dissipation of kinetic energy and $\mu$ is the apparent dense phase viscosity. Therefore, it seems reasonable to assume that a relationship exists between attrition and particle velocity gradients. Hence, in regions of high velocity gradient attrition will be higher. Fig. 14 shows the maximum value of $\sigma$ as a function of the total influence region.

Fig. 14 shows that the volumetric dissipation of kinetic energy is proportional to the square root of the total influence region. Fig. 13b shows a linear relationship between the total influence region, $A_{t}$, and the square of the equivalent bubble diameter. Therefore, the

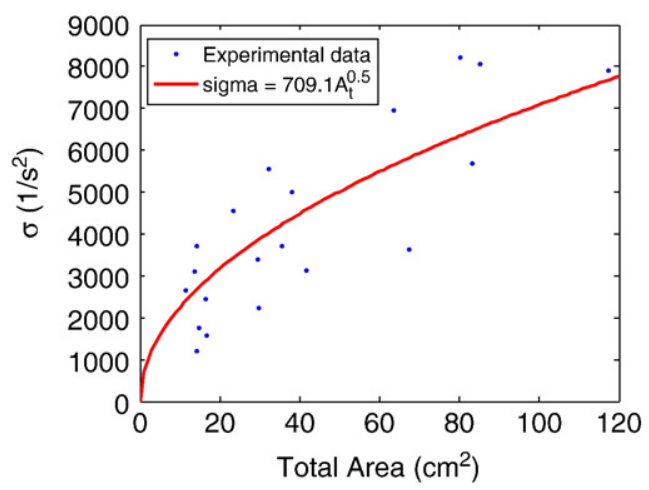

Fig. 14. $\sigma$ vs. total influence region previously calculated.

volumetric dissipation of kinetic energy is proportional to the equivalent diameter of the bubble: $\sigma \propto D_{e q}$. As the bubble velocity is related to the bubble diameter by the expression $U_{b}=\phi \sqrt{ } g D_{e q}[20]$, we can conclude that the volumetric dissipation of kinetic energy of the dense phase is related to the kinetic energy of a rising bubble: $\sigma \propto U_{b}^{2}$.

\section{Conclusions}

PIV and DIA techniques were employed in a 2 D fluidized bed in order to distinguish the dense phase from the bubble phase and obtain the time averaged velocity of the dense phase as well as the proportion of time that a region was occupied by a bubble. The perturbation in the dense phase induced by a rising bubble and the quantification of the area of influence of a bubble in the dense phase were obtained from the experimental results.

The present work has shown that there are three regions clearly defined around a bubble in terms of its velocity fluctuation: 1 ) a region above the bubble dome, 2) a region with an upward moving dense phase in which particles are transported into the drift of the bubble and 3 ) a region with a downwardly moving dense phase in which particles surround the bubble towards its wake. For coalescing bubbles, it was not possible to distinguish which regions corresponded to each bubble due to the interference between the wakes of the leading and the trailing bubbles. It is also shown that the velocity fluctuation of the dense phase diminishes sharply beyond the influence region. Hence, the horizontal derivative of the vertical velocity fluctuation around a bubble can be used to delimit the region of influence of a bubble. Using this procedure, the experimental results indicate that the area perturbed by the velocity of the dense phase is proportional to the square of the bubble diameter. As the ascent velocity of bubbles is related to their diameter, the region of influence of a bubble in the dense phase is linked to the dissipation of the bubble kinetic energy.

\section{Notation}

$A_{\mathrm{d}} \quad$ drift region, $\mathrm{cm}^{2}$

$A_{\mathrm{t}} \quad$ influence region, $\mathrm{cm}^{2}$

$C_{n}(\boldsymbol{x}) \quad$ dense phase $($ level $=1$ ) or bubble phase (level $=0$ ) for a point at position $\boldsymbol{x}$

$\overline{C(\boldsymbol{x})} \quad$ fraction of time that a point is occupied by dense phase

$d_{\mathrm{p}} \quad$ particle diameter, $\mathrm{cm}$

$f_{\mathrm{a}} \quad$ acquisition frequency, $\mathrm{Hz}$

$h \quad$ fluidized bed height, $\mathrm{cm}$

$h_{1} \quad$ recorded height of the fluidized bed, $\mathrm{cm}$

$M \quad$ mixing degree

$N \quad$ number of images selected for analysis

$t \quad$ fluidized bed thickness, $\mathrm{cm}$

$T$ time interval of the measurement, $\mathrm{s}$

$U_{\mathrm{g}} \quad$ superficial gas velocity, $\mathrm{cm} / \mathrm{s}$

$\overline{U(\boldsymbol{x})} \quad$ time averaged dense phase velocity, $\mathrm{cm} / \mathrm{s}$ 
$U_{\mathrm{b}} \quad$ bubble velocity, $\mathrm{cm} / \mathrm{s}$

$U_{\mathrm{y}} \quad$ vertical dense phase velocity, $\mathrm{cm} / \mathrm{s}$

$\boldsymbol{x}_{\mathrm{cm}} \quad$ center of mass coordinates of the bubble, $\mathrm{cm}$

$U(\boldsymbol{x}, t) \quad$ dense phase velocity, $\mathrm{cm} / \mathrm{s}$

$U^{\prime}(\boldsymbol{x}, t) \quad$ fluctuating velocity component, $\mathrm{cm} / \mathrm{s}$

$U_{\mathrm{mf}} \quad$ minimum fluidization velocity, $\mathrm{cm} / \mathrm{s}$

$w \quad$ fluidized bed width, $\mathrm{cm}$

$w_{1} \quad$ recorded width of the fluidized bed, $\mathrm{cm}$

$\boldsymbol{x} \quad$ horizontal and vertical coordinates for a point $x=x \vec{i}+y \overrightarrow{j,} \mathrm{~cm}$

\section{Greek letters}

$\mu \quad$ apparent dense phase viscosity, $\mathrm{kg} /(\mathrm{ms})$

$\rho_{\mathrm{p}} \quad$ particle density, $\mathrm{kg} / \mathrm{m}^{3}$

$\sigma \quad$ ratio between the volumetric dissipation of kinetic energy and the apparent viscosity, $1 / \mathrm{s}^{2}$

$\Phi_{v} \quad$ volumetric dissipation of kinetic energy, $\mathrm{kg} /\left(\mathrm{ms}^{3}\right)$

\section{References}

[1] P.M.C. Lacey, Developments in the theory of particle mixing, Journal of applied chemistry 4 (1954) 257-268.

[2] L. Shen, M. Zhang, Y. Xu, Solid mixing in fluidized beds, Powder Technology 84 (1995) 207-212.

[3] D. Kunii, O. Levenspiel, Fluidization Engineering, Wiley, New York, 1969.

[4] L.T. Fan, J.C. Song, N. Yutani, Radial particle mixing in gas-solids fluidized beds, Chemical Engineering Science 41 (1986) 117.

[5] L-S Lu and S-S Hsiau, Mixing in a vibrated granular bed: Diffusive and convective effects, Powder Technology 184 (2008) 31-43.

[6] S. Sánchez-Delgado, J.A. Almendros-Ibáñez, A. Soria-Verdugo, D. Santana, U. RuizRivas, Coherent structure and bubble-particle velocity in 2-D fluidized beds,
Proceeding of the 9th International Conference on Circulating Fluidized Beds, 2008, pp. 1007-1012.

[7] J.A. Almendros-Ibáñez, S. Sánchez-Delgado, C. Sobrino, D. Santana, Experimental observations on the different mechanisms for solid ejection in gas-fluidized beds, Chemical Engineering and Processing 48 (2009) 734-744.

[8] A. Busciglio, G. Vella, G. Micale, L. Rizzuti, Analysis of the bubbling behaviour of 2D gas solid fluidized beds Part I: digital image analysis technique, Chemical Engineering Journal 140 (2007) 398-413.

[9] J.A. Laverman, I. Roghair, M. Van Sint Annaland, H. Kuipers, Investigation into the hydrodynamics of gas-solid fluidized beds using particle image velocimetry coupled with digital image analysis, The Canadian Journal of Chemical Engineering 86 (2008) 523-535.

[10] Z. Liu, Y. Zheng, L. Jia, Q. Zhang, Study of bubble induced flow structure using PIV, Chemical Engineering Science 60 (2005) 3537-3552.

[11] J. Werther, J. Reppenhagen, Handbook of fluidization and fluid-particle system, in: Wen-Ching Yang (Ed.), Marcel Dekker Inc, New York, 2003, pp. 97-106.

[12] D. Geldart, Types of gas fluidization, Powder Technology 7 (1973) 285-292.

[13] F. Johnsson, R.C. Zijerveldb, J.C. Schoutenb, C.M. van den Bleek, B. Leckner, Characterization of fluidization regimes by time-series analysis of pressure fluctuations, International Journal of Multiphase Flow 26 (2000) 663-715.

[14] S. Sasic, F. Johnsson, B. Leckner, Interaction between a fluidized bed and its airsupply system: some observations, Industrial and Engineering Chemistry Research 43 (2004) 5730-5737.

[15] S. Sasic, B. Leckner, F. Johnsson, Fluctuations and waves in fluidized bed systems: the influence of the air-supply system. Powder Technology 153 (2005) 176-195.

[16] J. K. Sveen (1998-2007), http://www.math.uio.no// \{\}jks/matpiv.

[17] C.R. Müller, J.F. Davidson, J.S. Dennis, A.N. Hayhurst, A study of the motion and Eruption of a Bubble at the surface of a two-dimensional fluidized bed using particle image velocimetry (PIV), Industrial \& Engineering Chemistry Research 46 (2007) $1642-1652$.

[18] N. Otsu, A threshold selection method from gray-level histograms, IEEE Transactions on Systems, Man, and Cybernetics 9 (1979) 62-66.

[19] G.R. Caicedo, Juan J. Prieto Marqués, Mónica García Ruíz, Jesús Guardiola Soler, A study on the behaviour of bubbles of a 2D gas-solid fluidized bed using digital image analysis, Chemical Engineering and Processing 42 (2003) 9-14.

[20] J.F. Davidson, D. Harrison, Fluidised Particles, Cambridge at the University Press, 1963. 